\title{
Radiation dose in non-dental cone beam CT applications: a systematic review
}

\author{
Cosimo Nardi ${ }^{1}$ - Sergio Salerno ${ }^{2} \cdot$ Roberto Molteni $^{3} \cdot$ Mariaelena Occhipinti $^{4}$ - Giulia Grazzini ${ }^{1} \cdot$ Niccolò Norberti $^{1}$. \\ Cesare Cordopatri ${ }^{1} \cdot$ Stefano Colagrande ${ }^{1}$
}

Received: 13 February 2018 / Accepted: 24 May 2018 / Published online: 5 June 2018

(c) Italian Society of Medical Radiology 2018

\begin{abstract}
Background Radiation-induced health risks are broadly questioned in the literature. As cone beam computed tomography (CBCT) is increasingly used in non-dental examinations, its effective dose needs to be known. This study aimed to review the published evidence on effective dose of non-dental CBCT for diagnostic use by focusing on dosimetry system used to estimate dose.

Materials and methods A systematic review of the literature was performed on 12 November 2017. All the literature up to this date was included. The PubMed and web of science databases were searched. Studies were screened for inclusion based on defined inclusion and exclusion criteria according to the preferred reporting items for systematic reviews.

Results Fifteen studies met the inclusion criteria and were included in our review. Thirteen and two of them examined one and two anatomical areas, respectively. The anatomical areas were: ear (6), paranasal sinuses (4), ankle (3), wrist (2), knee (1), and cervical spine (1). Effective dose was estimated by different methods: (i) RANDO phantom associated with thermoluminescent dosimeters (6), metal oxide semiconductor field-effect transistor dosimeters (3), and optically stimulated luminescent dosimeters (1). (ii) Scanner outputs, namely computed tomography dose index (1) and dose area product (2). (iii) Monte Carlo simulations (2).

Conclusion CBCT of extremities, cervical spine, ears and paranasal sinuses was found to be a low-dose volumetric imaging technique. Effective doses varied significantly because of different exposure settings of CBCT-units and different dosimetry systems used to estimate dose.
\end{abstract}

Keywords Radiation dosage $\cdot$ Effective dose $\cdot$ Cone beam computed tomography $\cdot$ Head and neck imaging $\cdot$ Skeletal imaging

Stefano Colagrande

stefano.colagrande@unifi.it

Cosimo Nardi

cosimo.nardi@unifi.it

Sergio Salerno

sergio.salerno@unipa.it

Roberto Molteni

robertodocmolteni@yahoo.com

Mariaelena Occhipinti

mariaelena.occhipinti@unifi.it

Giulia Grazzini

grazzini.giulia@gmail.com

Niccolò Norberti

norberti.niccolo@gmail.com

Cesare Cordopatri

cordopatri.cesare@gmail.com
1 Department of Experimental and Clinical Biomedical Sciences, Radiodiagnostic Unit n. 2, University of Florence - Azienda Ospedaliero-Universitaria Careggi, Largo Brambilla 3, 50134 Florence, Italy

2 Department of Radiology, University Hospital Paolo Giaccone of Palermo, Via del Vespro 127, 90127 Palermo, Italy

3 Skanray Europe, Via Cicogna, 38, 40068 San Lazzaro di Savena, Bologna, Italy

4 Department of Experimental and Clinical Medicine, Section of Respiratory Medicine, University of Florence - Azienda Ospedaliero-Universitaria Careggi, Largo Brambilla 3, 50134 Florence, Italy 


\section{Introduction}

Cone beam computed tomography (CBCT) is a volumetric imaging technique extensively used and well established in all areas of dental diagnostics [1-5]. Recently, it is increasingly used in the study of ears, paranasal sinuses, and extremities [6-8]. Numerous manufacturers today offer a wide range of CBCT-units with technical features which differ greatly [9]. There are horizontal and vertical units that examine patients in supine, seated, or upright positions. CBCT devoted to the study of ears and paranasal sinuses or designed to assess the extremities in weightbearing position (i.e. under load) have newly entered the market as well [10].

The success of CBCT is mainly due to a relatively low radiation dose [11], limited metal artefacts [12], high spatial resolution (0.075-0.4 mm isotropic voxel) [13], and low maintenance and operating costs [14]. Nevertheless, CBCT is not used for contrast-enhanced examinations, does not have a high contrast resolution [15], and the scan time is long with non-negligible motion artefacts [16].

$\mathrm{X}$-ray imaging for diagnostic purposes has gradually increased, which may have led to an increase in radiationinduced health risks [17-19]. For this reason, both in the USA (Senate Bill 1237) and Europe (Euratom directive 59/2013) legislations have been published that intensely request from operators to record the estimated patient dose of each radiation exposure in every radiology report $[20,21]$. This may be useful not only for estimating the potential risk of radiation exposure but also for assessing protocol optimisation, standardisation, and quality assurance [22].

Currently, effective dose is the most commonly metric used to track patient dose and represents the stochastic health risk to the whole body, which is the probability to induce cancer and/or genetic damage from ionising radiation. It involves comparing dosimetric values from different examinations and modalities [23].

Radiation dose of CBCT in dental use was already discussed by different papers [24]. However, no review on the field of dosimetry of the latest non-dental CBCT applications has been carried out. Therefore, the purpose of this study was to investigate the existing literature concerning the effective dose of non-dental CBCT used for diagnostic aims. An additional purpose is to analyse the methods used to measure and estimate the effective dose.

\section{Materials and methods}

\section{Literature searches}

The literature review was carried out in conformity with the preferred reporting items for systematic reviews (PRISMA) statement for studies focused on the dosimetric evaluation of CBCT, except in dental applications. PRISMA is an evidence-based minimum set of items that helps authors to improve the reporting of systematic reviews, although it is not a quality assessment tool to estimate the merit of a research [25].

The search strategy was restricted to English-language papers via the PubMed and web of science databases. The following combined terms were investigated: radiation dosage, radiation protection, dose, effective dose, absorbed dose, and cone beam computed tomography (Table 1). For the aims of this review, these terms were defined as follows:

- Radiation dosage and radiation protection according to medical subject headings (MeSH).

- Dose a generic term including all kinds of doses described by the International Commission on Radiological Protection (ICRP) [26].

- Effective dose the sum of the products of the tissue weighting factors and the absorbed dose within the exposed tissues and organs of the body-established by the ICRP.

- Absorbed dose the quantity of ionising radiation absorbed by a body, measured as the energy absorbed per unit mass-established by the ICRP.

- Cone beam computed tomography a volumetric imaging technique with a conic/pyramidal X-ray beam of radiation.

The search in Pubmed included both MeSH and freetext terms, whereas the searches in Web of Science included only free-text terms. An additional manual search was performed using the reference lists of the examined studies. The searches were conducted on 12 November 2017.

\section{Inclusion and exclusion criteria}

We included studies published in international peer-reviewed journals that examined effective dose of non-dental CBCT for diagnostic use. Anatomical areas in the facial region which can have a relationship with tooth, such as temporomandibular joints, nose and sinonasal cavities were included. Original articles, case reports, short communications, letters to the editor, and conference proceedings were included. 
Table 1 Search strategy

\begin{tabular}{lr}
\hline Indexing terms & Publications $(N)$ \\
\hline Pubmed & \\
\#01 Radiation dosage [MeSH terms] & 76,952 \\
\#02 Radiation dosage & 128,749 \\
\#03 Radiation protection [MeSH terms] & 19,449 \\
\#04 Radiation protection & 36,398 \\
\#05 Dose & $1,236,797$ \\
\#06 Effective dose & 144,960 \\
\#07 Absorbed dose & 16,388 \\
\#08 Cone beam computed tomography [MeSH & 6520 \\
terms] & \\
\#09 Cone beam computed tomography & 9875 \\
\#10=\#01 OR \#02 OR \#03 OR \#04 OR \#05 OR & $1,281,659$ \\
\#06 OR \#07 & \\
\#11 =\#08 OR \#09 & 9875 \\
\#12=\#10 AND \#11 & 2136 \\
Web of science & \\
Dose & \\
AND & \\
Cone beam computed tomography & \\
NOT & \\
Dental & \\
\end{tabular}

The exclusion criteria were:

- anything focusing on teeth;

- papers that did not focus on CBCT;

- papers that focused on CBCT for non-diagnostic purposes (e.g. guidance for radiotherapy);

- papers that did not relate to dose;

- papers in the field of dosimetry that did not estimate the effective dose.

\section{Study selection and data extraction}

Three reviewers independently examined the titles and abstracts of studies to determine their eligibility for inclusion. Screening the full text was done whenever the abstract did not give enough information to define eligibility. Moreover, the full text was read when at least one of the authors claimed that the study met the inclusion criteria. Data were individually extracted from each study on (1) the type of endpoint, (2) the anatomical area, (3) the physical and technical features of CBCT-units, especially scanning protocols, (4) the method to measure and estimate radiation dosage, (5) the features of dosimeters and phantoms, and (6) the effective dose.

In case of disagreement over the study selection or data extraction, the issue was solved by consensus discussion among the reviewers.

\section{Results}

\section{Study selection}

Fifteen studies met our inclusion criteria. Each stage of the search and screening processes with the number of papers identified, included, and excluded is shown in Fig. 1. The screening of the found articles, based on title and abstract reading, revealed 27 papers which were potentially eligible and consequently selected for full-text reading. After examination of the full texts, 12 papers did not meet the inclusion criteria. The main reasons for exclusion were that the studies did not focus on the field of dosimetry or did not report the effective dose. Among the 15 papers selected (14 original papers [27-40] and 1 conference proceeding [41]), two of them analysed two anatomical areas; all the others analysed only one anatomical area (Table 2). The following anatomical areas were analysed: ear [27-32], paranasal sinuses [27, 33, 34, 41], ankle [35-37], wrist [38, 39], knee [40], and cervical spine [32].

\section{Features of CBCT-units}

In the 15 studies examined, 16 CBCT-units (seven different models) were used, of which five were from the USA [29, 33, 34, 36, 41], Finland [31, 35, 37, 38, 40], and Italy $[27,28,32,35,39]$, and the remaining one from Japan [30]. One study [31] used a modified specific acquisition system that involved a pause during each exposure of the multiple frames to reduce potential motion artefacts generated by the rotating "C" arm. No physical/technical parameter was referred to in the three studies [34, 37, 41]. The tube voltage was mentioned in all the other studies, whereas some of them did not report field of view (FOV) $[28,29]$, mAs [30, 38, 39], and voxel size [27, 28, 40].

\section{Features of dosimetry systems for calculating effective dose}

The studies analysed in the current review measured or estimated the effective dose by using the following methods (Table 3):

- RANDO Phantom associated with three different kinds of dosimeters thermoluminescent dosimeter (TLD) [27-29, 32, 33, 41], metal oxide semiconductor fieldeffect transistor (MOSFET) dosimeter [31, 35, 40], and optically stimulated luminescent dosimeter (OSLD) [36]. The features of dosimeters and phantoms were generally described in detail. 
Fig. 1 Flowchart consistent with preferred reporting items for systematic reviews (PRISMA) statement

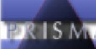

PRISMA 2009 Flow Diagram
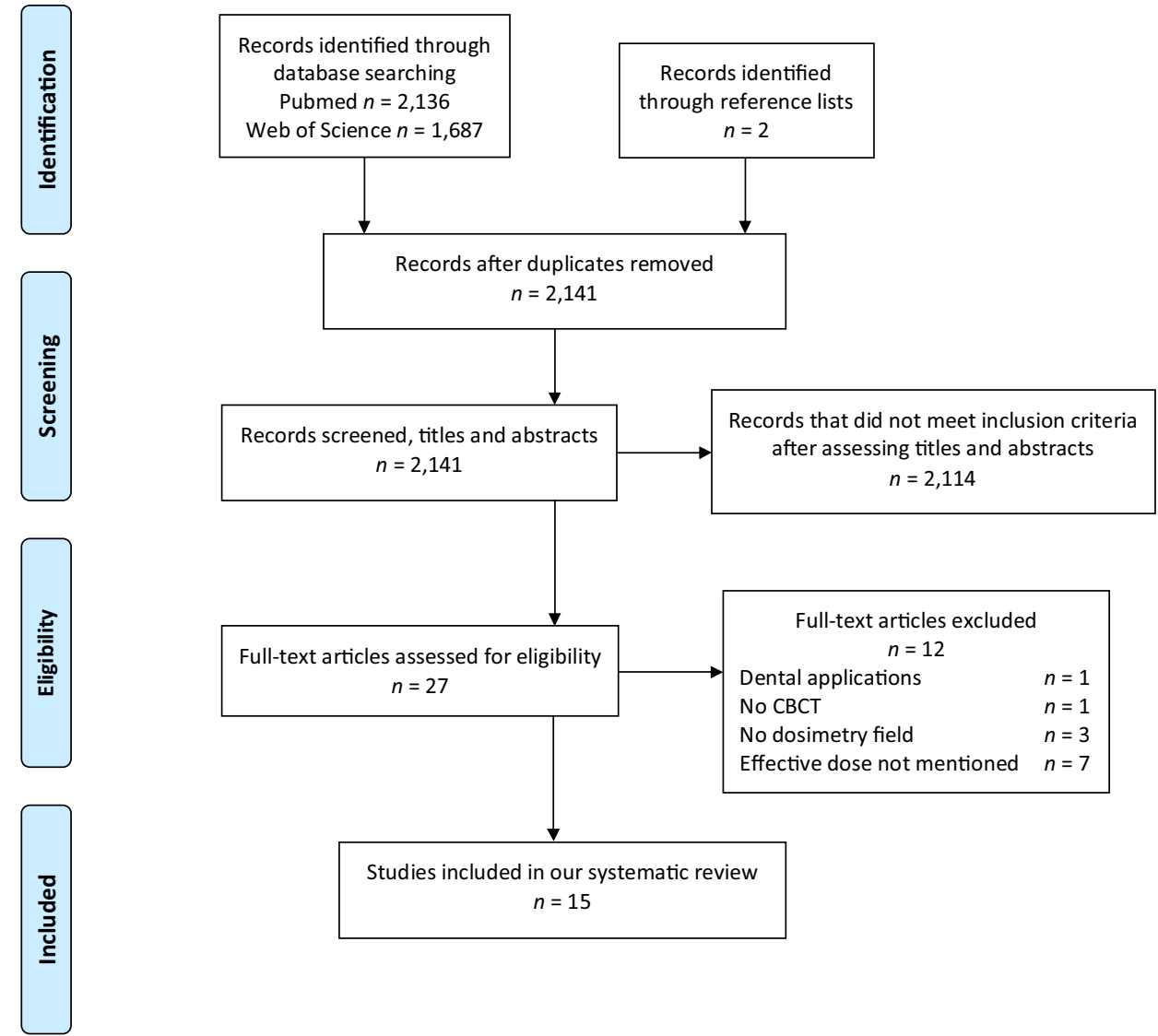

Studies included in our systematic review $n=15$

\section{Discussion} (CTDI) [39] and dose area product (DAP) [37, 38], each of which was multiplied by a conversion factor.

- Monte Carlo simulations [27, 34].

One study [27] compared the effective dose calculated by Monte Carlo simulations and the effective dose measured by TLD, while another [30] did not even mention the method used to calculate the effective dose.

In addition, the dosimetric study was the primary endpoint in 10 studies [27, 28, 31-36, 40, 41] and the secondary endpoint in 5 studies [29, 30, 37-39].

Finally, although it is beyond the purpose of the review, two studies [32, 33] provided a quantitative assessment of image quality. They are the only two studies published in 2017. All the others are older.
The large variability in both the physical/technical features of the CBCT-units-especially tube voltage, tube current, exposure time, and FOV - and the different methods used to measure or estimate the effective dose produced significant differences in the dosimetric values, thus making analysis of the results somewhat difficult. Furthermore, since the description of the exposure parameters and scanning protocols was often incomplete, realising the reasons for the different results on the effective dose was hard.

\section{Analysis of dosimetry systems for calculating the effective dose}

TLDs inserted in a RANDO phantom represent the conventional reference method for assessing the effective dose. Nevertheless, TLDs need to be replaced within the phantom after every exposure. 


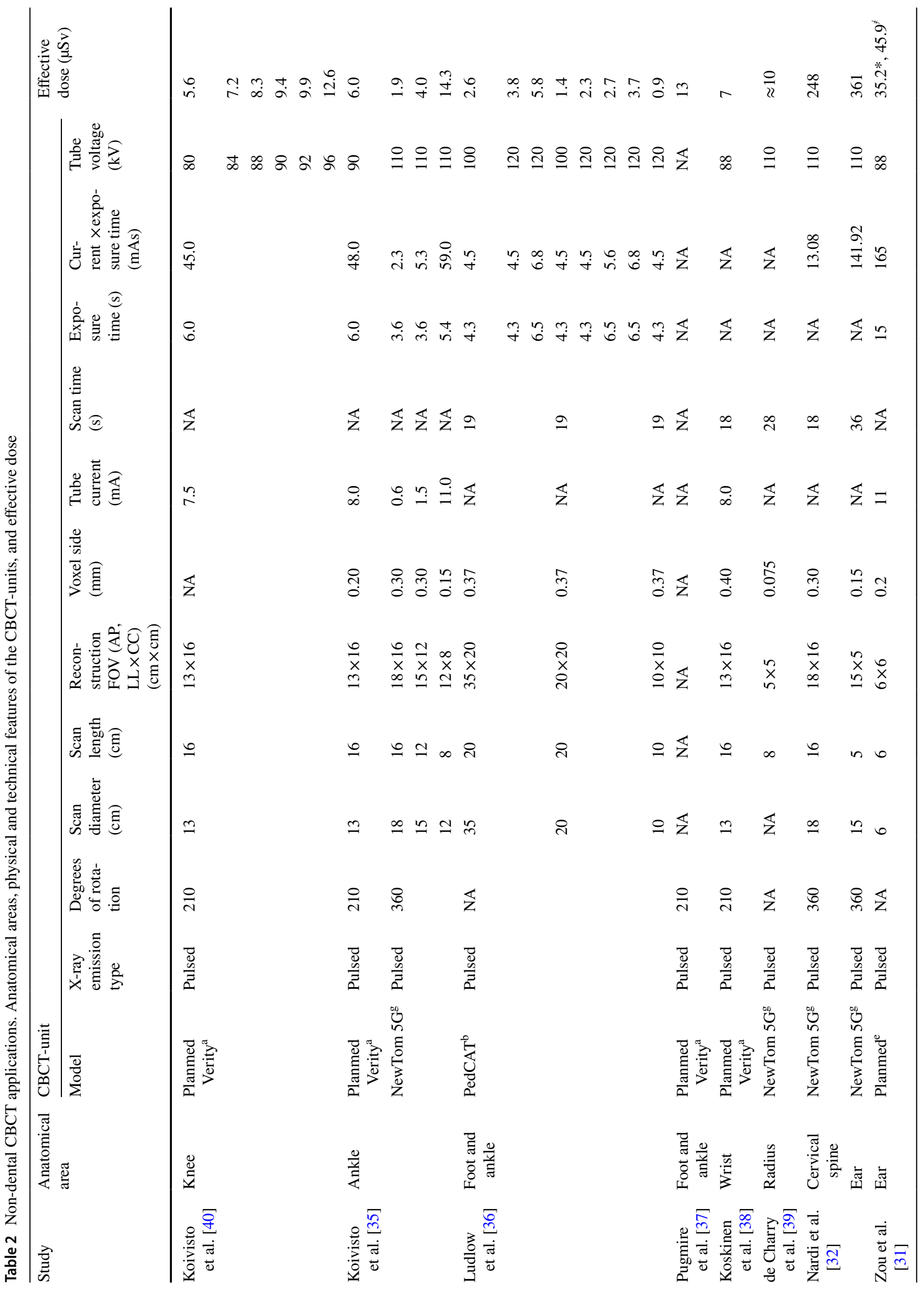




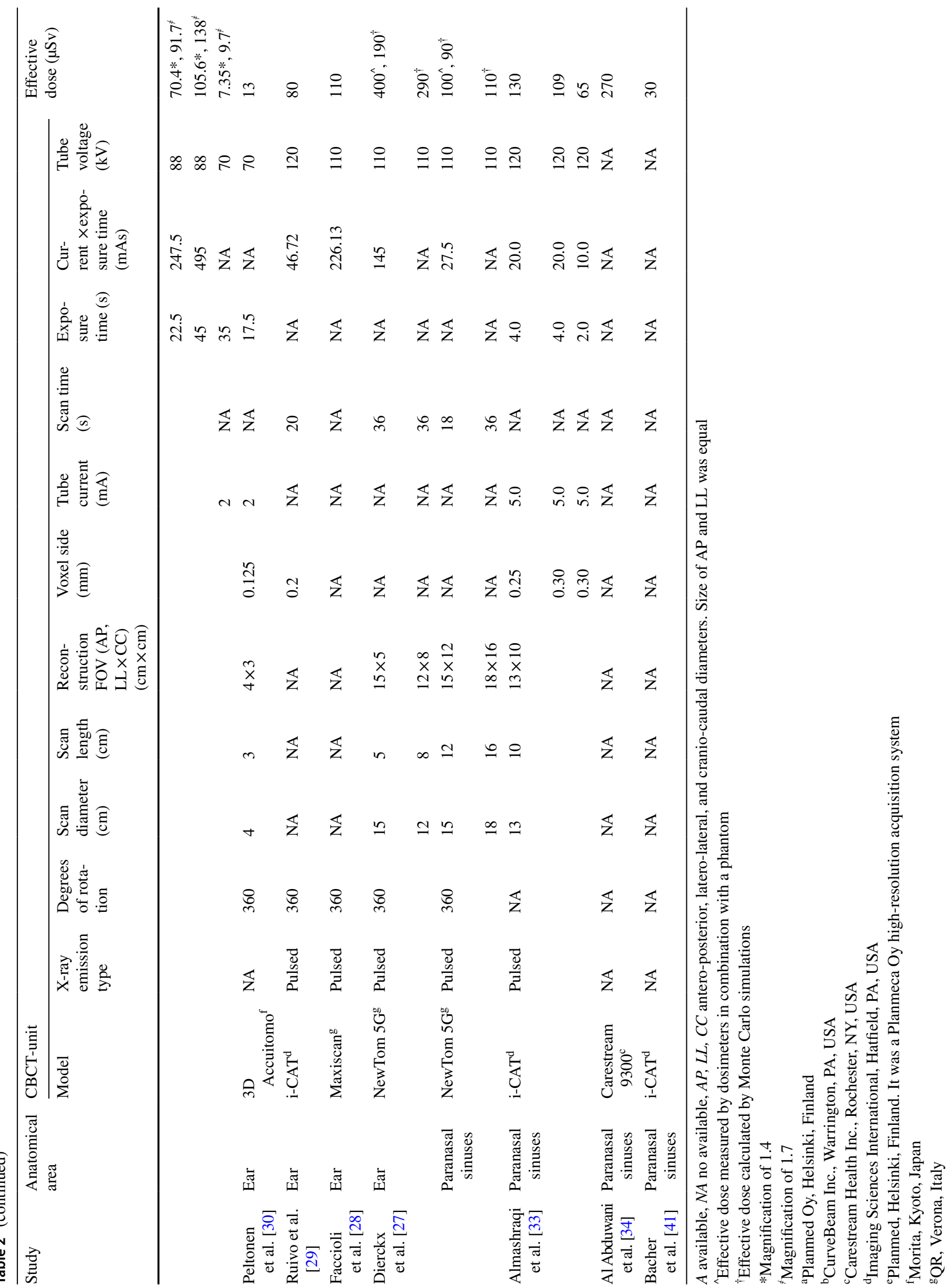


Since several exposures are recommended to attain more reliable dosimetric values, measuring the effective dose by TLD is very laborious, time-consuming, and prone to mistakes. To overcome these limitations, MOSFET dosimeters have been introduced lately. MOSFETs are purely electronic dosimeters connected to a reader that transfers data to a computer. They can perform multiple real-time measurements without having to repeatedly disassemble and reposition the phantom. The drawback of MOSFETs is that they are visible on the radiographs and produce a heel effect $[42,43]$.

OSLDs consist of crystals whose electrons collect the energy released from X-rays. At the dosimeter readout, the crystal is stimulated with a light-emitting diode, allowing the electrons to fall back to their original energy state while emitting a characteristic light proportional to the amount of the absorbed radiation dose. OSLDs have a small size and can be positioned on the patient skin. Although several considerations and conditions-such as beam energy and spectrum, dose range, geometrical arrangement, and reading protocol-theoretically warrant the use of specific correction factors, these factors and the related uncertainties are mostly in the single-digit per cent in the typical conditions of medical diagnostic CT. The most significant practical correction factor (easily determinable) may be the one to account for the beam average $\mathrm{kV}$. Contrary to the perception by some, signal fading is not a significant issue over a period of several days or weeks if the OSLDs are handled properly [44, 45].

CTDI and DAP are radiation dose outputs that indicate the amount of radiation directed towards the patient and allow one to compare the radiations of different CT systems. Three papers [37-39] under the current review used a scanner output to calculate the effective dose of extremities by using conventional X-ray conversion factors. CTDI calculates the dose by a pencil ionisation chamber that is only $100 \mathrm{~mm}$ long. Therefore, it neither detects the scattered dose over this length nor collects the primary beam in CBCT with a large FOV [46]. The DAP measures the radiation dose to air (without backscatter) multiplied by the area of the X-ray field.

The Monte Carlo simulation is a software-based technique used to simulate photon interactions with living matter since it tracks the trajectories of each individual photon and calculates the amount of energy released point by point. Monte Carlo algorithms are very demanding in computational resources and may require a long time to be performed (depending on the computing power). Although up to $10^{9}$ photons can typically be simulated with the computing power of current hardware and software resources, $\mathrm{CT}$ examinations involve a larger number of photons (up to $10^{16}$ photons). This may limit the accuracy of the dose estimations [47]. The only paper [27] which compared Monte
Carlo simulations and TLD, while keeping unchanged all CBCT-unit scanning parameters, found that Monte Carlo simulations underestimated the effective dose by more than $50 \%$ in the ears study and by $10 \%$ in the paranasal sinuses study, with respect to TLD.

\section{Radiation dose of extremities and cervical spine}

\section{Knee}

Koivisto et al. [40] used MOSFET dosimeters inserted in a custom-made RANDO phantom. They did not alter the exposure settings, except for the tube voltage, which was changed from 80 to $96 \mathrm{kV}$ with a proportionate increase in the effective dose from 5.6 to $12.6 \mu \mathrm{Sv}$.

\section{Ankle}

Koivisto et al. [35] and Ludlow et al. [36] used three different CBCT-units and two different dosimeters (MOSFET e OSLD) inserted in a custom-made RANDO phantom. Seven different FOVs with variable exposure time, $\mathrm{mAs}$, and $\mathrm{kV}$ resulted in effective doses from 0.9 to $14.3 \mu \mathrm{Sv}$. Pugmire et al. [37] calculated the effective dose of $13 \mu \mathrm{Sv}$ in the paediatric human ankle using the DAP-to-effective dose conventional X-ray conversion factors [48]; however, no CBCT-unit physical/technical parameter was reported.

\section{Wrist}

Koskinen et al. [38] and de Charry et al. [39] calculated effective doses of $7 \mu \mathrm{Sv}$ and approximately $10 \mu \mathrm{Sv}$ in adult human wrist and cadaveric distal radius using the DAP- and CTDI-to-effective dose wrist conventional X-ray conversion factors [49], respectively.

The overall mean effective dose for the studies on the extremities was $7.1 \mu \mathrm{Sv}$.

\section{Cervical spine}

Nardi et al. [32] measured the effective dose of $248 \mu \mathrm{Sv}$ by using TLDs in combination with an Alderson-RANDO phantom.

\section{Radiation dose of ear and paranasal sinuses}

\section{Ear}

Dierckx et al. [27], Nardi et al. [32], Faccioli et al. [28], and Ruivo et al. [29] examined the ear by using TLDs inserted in an Alderson-RANDO phantom. The resulting effective doses were $400,361,110$, and $80 \mu \mathrm{Sv}$, respectively. The 


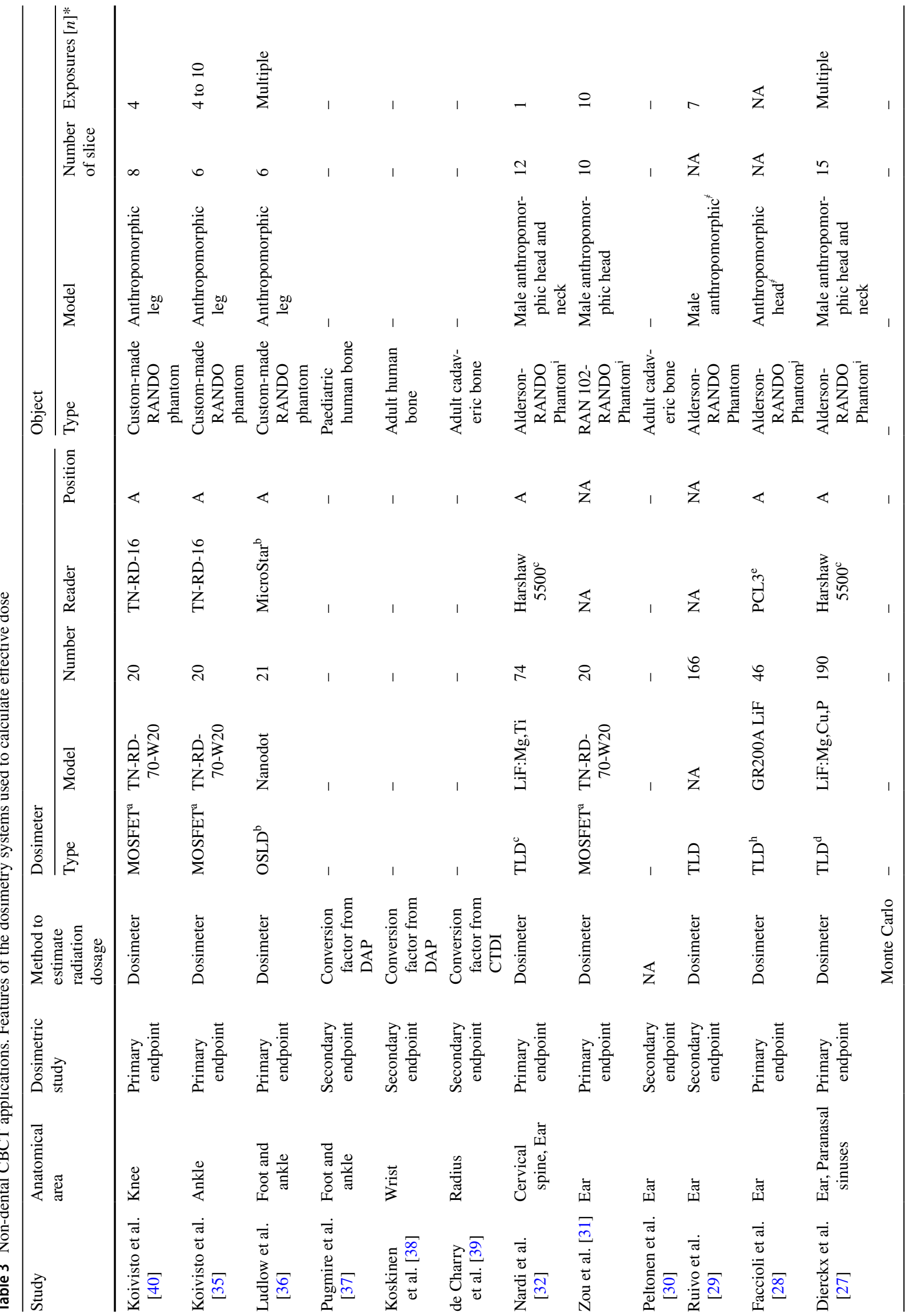




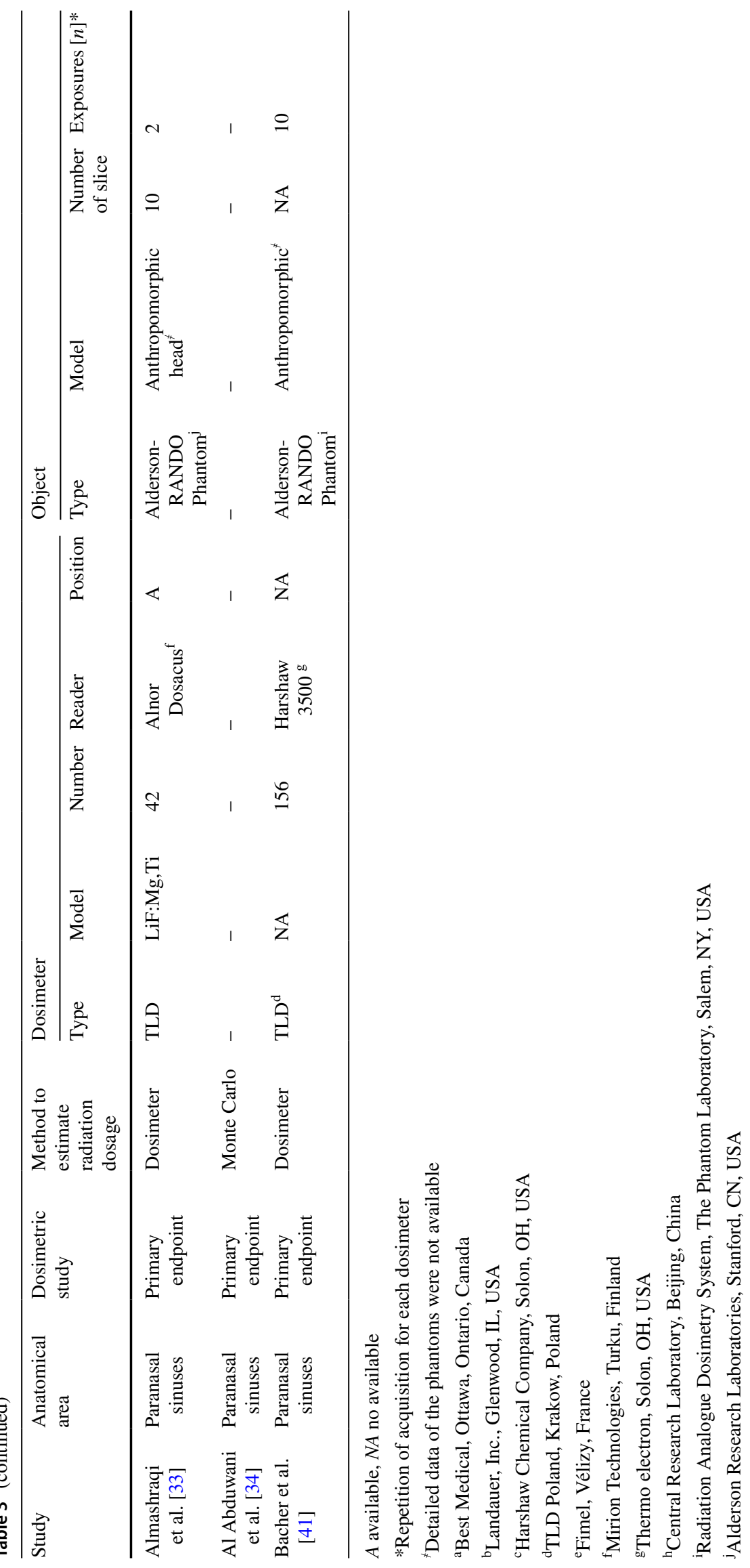


first two aforementioned authors [27, 32] used a large FOV including both ears, whereas the other two authors [28, 29] did not mention the size of the FOV. Dierckx et al. [27] also used Monte Carlo simulations, obtaining an effective dose that was less than half compared to the effective dose measured by TLD. Zou et al. [31] used MOSFET dosimeters and a small FOV; they changed the exposure time and found that the effective dose varied from 7 to $138 \mu \mathrm{Sv}$. Peltonen et al. [30] reported an effective dose of $13 \mu \mathrm{Sv}$ in the cadaveric temporal bone without specifying the dosimetry system and adapting a dental CBCT-unit with a small FOV.

The overall mean effective dose for the studies on the ear was $178.2 \mu \mathrm{Sv}$.

\section{Paranasal sinuses}

Dierckx et al. [27] measured the effective dose via a specific FOV $(15 \times 12 \mathrm{~cm})$ for the paranasal sinuses study using both TLDs inserted in an Alderson-RANDO phantom and Monte Carlo simulations. The effective dose was 100 and $90 \mu \mathrm{Sv}$, respectively. They also estimated the effective dose of $110 \mu \mathrm{Sv}$ using a larger FOV $(18 \times 16 \mathrm{~cm})$ by Monte Carlo simulations. Almashraqi et al. [33] investigated the paranasal sinuses by TLDs inserted in an Alderson-RANDO phantom. They used a slightly smaller FOV $(13 \times 10 \mathrm{~cm})$ than Dierckx, and changed both the voxel size $(0.25$ or $0.30 \mathrm{~mm})$ and the exposure time ( 2 or $4 \mathrm{~s}$ ). The effective dose varied from 65 to $130 \mu \mathrm{Sv}$. Al Abduwani et al. [34] and Bacher et al. [41] estimated effective doses of 270 and $30 \mu \mathrm{Sv}$ by using Monte Carlo simulations and TLDs, respectively, without mentioning any protocol parameter. The overall mean effective dose for the studies on the paranasal sinuses was $119.0 \mu \mathrm{Sv}$.

Among all the aforementioned authors, only Nardi et al. [32] and Almashraqi et al. [33] provided a quantitative assessment of image quality that represents a key element which should be associated with a dosimetric study. In fact, each physical/technical parameter of every CBCT-unit must be optimised in order to limit the exposure, reach a diagnostically acceptable image quality in relation to the clinical query, and allow the comparison of doses using different imaging techniques.

The ear study requires a high resolution to detect the tiny structures of which it is constituted; consequently, a high radiation dosage is needed [32]. The dose can be generally reduced in the paranasal sinuses, cervical spine, and extremities studies, where the image quality is adequate even with low-dose protocols [32, 33, 50]. Low-dose protocols should be set in all imaging techniques with ionising radiation, especially in CBCT, because of several units with extremely variable acquisition parameters and therefore very different exposures, as shown in the current review. The parameter optimisation has already been discussed in dental CBCT, but further research shall be carried out in non-dental CBCT applications. Furthermore, although CBCT is considered to be a low-dose volumetric imaging technique, in clinical practice the repetition rate of examinations due to motion artefacts is not insignificant $(0.9-5.4 \%)$ [12, 51, 52]; this increases the mean radiation dose administered to patients.

Overall, the mean CBCT effective dose for the extremities $(7.1 \mu \mathrm{Sv})$ was a little more than double and seven times the amount of one projection X-ray effective dose for the knee $(3.0 \mu \mathrm{Sv})[40]$ and the foot-ankle $(1.0 \mu \mathrm{Sv})$ region [35, 36], respectively. The study of extremities by radiographs needs more than one plain film, and a bone fracture is not always detectable by two-dimensional medical imaging because of the geometric distortion effect and the superimposition of three-dimensional complex skeletal structures $[53,54]$. Therefore, we suppose that CBCT may sometimes replace a conventional $\mathrm{X}$-ray examination in extremity bone trauma turned into a first-level imaging since CBCT assures high diagnostic accuracy in the detection of bone fractures [55-58] with an acceptable radiation dose.

Using a standard RANDO phantom or a computational patient model has important limitations because neither system takes into account the constitutional nature of any individual patient and the wide range of scan protocols. It is incorrect to employ a one-size-fits-all phantom to accurately estimate the dose to all patients. In fact, a typical adult phantom does not represent an individual patient-specific habitus; consequently, it will underestimate the dose for a paediatric patient and overestimate the dose for an obese patient because the X-ray beam is attenuated to a greater extent in large patients than in small patients [59].

We noticed that the physical/technical parameters necessary to make the value of an effective dose meaningful were reported more thoroughly in the studies in which the primary endpoint was represented by a dosimetric study. We firmly believe that an accurate report of the effective dose must always be combined with a detailed description of both the physical/technical parameters of any unit and the dosimetry system adopted. Otherwise, the value of the effective dose has poor relevance as it is not reproducible. Similarly, reporting only the scanner output value is not appropriate since scanner outputs are not a real measurement of the patient dose, not unless they are combined with the conversion factors, taking into account the patient's size/age/gender, irradiated organ, body composition, scan range, mAs, and tube voltage [60-62].

Currently, there are no conversion factors for non-dental CBCT. Therefore, using dental CBCT conversion factors is technically inaccurate since they concern a different anatomical area with different tissues and organs irradiated. Even more so, it is debatable to use the conversion factors of different technology systems as conventional X-rays or 
multislice CT. For that reason, future investigations on non-dental CBCT conversion factors are recommended.

We wonder whether it is proper to report the scanner output value for the estimation of patient dose in the radiology report as required by the latest international provisions. Scanner outputs do not take into account of variability in tissue weights; therefore, they are an unreliable surrogate for patient dose. We think that mentioning the scanner output value in the radiology report is just the first step towards monitoring both the exposure to the population and the dose to the individual patient. It is desirable for the future to achieve a fast, easy, accurate, cost-effective, and unambiguous system for estimating the effective dose since dosimeters cannot be placed within the organs of a human being. The ideal solution to ensure reliable patient dose measurements would be to have every CTunit displaying the effective dose directly on the computer screen at the end of each examination by innovative software Monte Carlo simulation-like or reliable conversion factors in combination with scanner outputs. This is a hard challenge for research.

The main limitation of our study was the impossibility of making a meta-analysis because of both the heterogeneity of the methods used to estimate the dose and the limited papers available on the various non-dental CBCT applications. A further limitation was discarding all papers that did not relate to the effective dose, even though they were related to dosimetry. Furthermore, although effective dose represents the most reliable metre used to track the dose for individual patients, effective dose is believed not to be an excellent parameter of patient risk per se [63-65] and the stochastic radiation damage may be underestimated [66, 67].

\section{Conclusions}

The current review proved that CBCT of the extremities, cervical spine, ears, and paranasal sinuses was a low-dose volumetric imaging technique. Nevertheless, the effective dose varied considerably among authors due to both the large number of different exposure settings of the several CBCT-units and the various dosimetry systems represented by scanner outputs, Monte Carlo simulations, and different kinds of dosimeters associated with RANDO phantom. Therefore, further studies are required to make the measurement methods uniform with more reliable and consistent estimations of the effective dose values.

\section{Compliance with ethical standards}

Conflicts of interest The authors declare that they have no conflict of interest.
Ethical standards This article does not contain any studies with human participants or animals performed by any of the authors.

\section{References}

1. Ahmad M, Jenny J, Downie M (2012) Application of cone beam computed tomography in oral and maxillofacial surgery. Aust Dent J 57:82-94

2. Bornstein MM, Horner K, Jacobs R (2017) Use of cone beam computed tomography in implant dentistry: current concepts, indications and limitations for clinical practice and research. Periodontology 73:51-72

3. Nardi C, De Falco L, Selvi V, Lorini C, Calistri L, Colagrande S (2018) Role of cone beam computed tomography with a large field of view in Goldenhar syndrome. Am J Orthod Dentofac Orthop 153:269-277

4. Chang E, Lam E, Shah P, Azarpazhooh A (2016) Cone-beam computed tomography for detecting vertical root fractures in endodontically treated teeth: a systematic review. J Endod 42:177-185

5. Sironi E, Taroni F, Baldinotti C, Nardi C, Norelli GA, Gallidabino M, Pinchi V (2018) Age estimation by assessment of pulp chamber volume: a Bayesian network for the evaluation of dental evidence. Int J Legal Med. https://doi.org/10.1007/s0041 4-017-1733-0

6. Nguyen TD, Kösling S, Mlynski R, Plontke SK (2016) Visualisation of passive middle ear implants by cone beam and multidetector computed tomography: a comparative in vitro study. Eur Radiol 26:4538-4544

7. Parks ET (2014) Cone beam computed tomography for the nasal cavity and paranasal sinuses. Dent Clin North Am 58:627-651

8. Nardi C, Buzzi R, Molteni R, Cossi C, Lorini C, Calistri L, Colagrande S (2017) The role of Cone Beam CT in the study of symptomatic total knee arthroplasty (TKA): a 20 cases report. Br J Radiol 90:20160925

9. Nemtoi A, Czink C, Haba D, Gahleitner A (2013) Cone beam CT: a current overview of devices. Dentomaxillofac Radiol 42:20120443

10. Thawait GK, Demehri S, AlMuhit A et al (2015) Extremity cone-beam CT for evaluation of medial tibiofemoral osteoarthritis: Initial experience in imaging of the weight-bearing and non-weight-bearing knee. Eur J Radiol 84:2564-2570

11. Ludlow JB, Timothy R, Walker C, Hunter R, Benavides E, Samuelson DB, Scheske MJ (2014) Effective dose of dental CBCT - a meta analysis of published data and additional data for nine CBCT units. Dentomaxillofac Radiol 15:20140197

12. Nardi C, Borri C, Regini F, Calistri L, Castellani A, Lorini C, Colagrande S (2015) Metal and motion artifacts by cone beam computed tomography (CBCT) in dental and maxillofacial study. Radiol Med 120:618-626

13. Watanabe H, Honda E, Tetsumura A, Kurabayashi T (2011) A comparative study for spatial resolution and subjective image characteristics of a multi-slice CT and a cone-beam CT for dental use. Eur J Radiol 77:397-402

14. Christell H, Birch S, Hedesiu M et al (2012) Variation in costs of cone beam CT examinations among healthcare systems. Dentomaxillofac Radiol 41:571-577

15. Lechuga L, Weidlich GA (2016) Cone beam CT vs. fan beam CT: a comparison of image quality and dose delivered between two differing CT imaging modalities. Cureus 8:778

16. Nardi C, Molteni R, Lorini C, Taliani GG, Matteuzzi B, Mazzoni E, Colagrande S (2016) Motion artefacts in cone beam CT: 
an in vitro study about the effects on the images. Br J Radiol 89:20150687

17. Fazel R, Krumholz HM, Wang Y et al (2009) Exposure to lowdose ionizing radiation from medical imaging procedures in the United States. N Engl J Med 361:849-857

18. Hendee WR, O'Connor MK (2012) Radiation risks of medical imaging: separating fact from fantasy. Radiology 264:312-321

19. Colagrande S, Origgi D, Zatelli G, Giovagnoni A, Salerno S (2014) CT exposure in adult and paediatric patients: a review of the mechanisms of damage, relative dose and consequent possible risks. Radiol Med 119:803-810

20. California Senate Bill 1237. http://www.leginfo.ca.gov/pub/0910/bill/sen/sb_1201-1250/sb_1237_bill_20100929_chaptered. html. Accessed Feb 2018

21. Council Directive 2013/59/Euratom of 5 December 2013 laying down basic safety standards for protection against the dangers arising from exposure to ionising radiation, and repealing Directives 89/618/Euratom, 90/641/Euratom, 96/29/Euratom, 97/43/Euratom and 2003/122/Euratom. http://data.europa.eu/ eli/dir/2013/59/oj. Accessed Feb 2018

22. Saltybaeva N, Jafari ME, Hupfer M, Kalender WA (2014) Estimates of effective dose for CT scans of the lower extremities. Radiology 273:153-159

23. McCollough CH, Leng S, Yu L, Cody DD, Boone JM, McNittGray MF (2011) CT dose index and patient dose: they are not the same thing. Radiology 259:311-316

24. Al-Okshi A, Lindh C, Salé H, Gunnarsson M, Rohlin M (2015) Effective dose of cone beam CT (CBCT) of the facial skeleton: a systematic review. Br J Radiol 88:20140658

25. Moher D, Liberati A, Tetzlaff J, Altman DG, PRISMA Group (2009) Preferred reporting items for systematic reviews and meta-analyses: the PRISMA statement. J Clin Epidemiol 62:1006-1012

26. Valentin J (2007) International Commission on Radiological Protection. Recommendations of the International Commission on Radiological Protection. ICRP Publication 103. Ann ICRP 37. Elsevier

27. Dierckx D, Saldarriaga Vargas C, Rogge F, Lichtherte S, Struelens L (2015) Dosimetric analysis of the use of CBCT in diagnostic radiology: sinus and middle ear. Radiat Prot Dosim 163:125-132

28. Faccioli N, Barillari M, Guariglia S, Zivelonghi E, Rizzotti A, Cerini R, Mucelli RP (2009) Radiation dose saving through the use of cone-beam CT in hearing-impaired patients. Radiol Med 114:1308-1318

29. Ruivo J, Mermuys K, Bacher K, Kuhweide R, Offeciers E, Casselman JW (2009) Cone beam computed tomography, a low-dose imaging technique in the postoperative assessment of cochlear implantation. Otol Neurotol 30:299-303

30. Peltonen LI, Aarnisalo AA, Kortesniemi MK, Suomalainen A, Jero J, Robinson S (2007) Limited cone-beam computed tomography imaging of the middle ear: a comparison with multislice helical computed tomography. Acta Radiol 48:207-212

31. Zou J, Koivisto J, Lähelmä J, Aarnisalo A, Wolff J, Pyykkö I (2015) Imaging optimization of temporal bones with cochlear implant using a high-resolution cone beam ct and the corresponding effective dose. Ann Otol Rhinol Laryngol 124:466-473

32. Nardi C, Talamonti C, Pallotta S, Saletti P, Calistri L, Cordopatri C, Colagrande S (2017) Head and neck effective dose and quantitative assessment of image quality: a study to compare cone beam CT and multislice spiral CT. Dentomaxillofac Radiol 46:20170030

33. Almashraqi AA, Ahmed EA, Mohamed NS, Barngkgei IH, Elsherbini NA, Halboub ES (2017) Evaluation of different lowdose multidetector $\mathrm{CT}$ and cone beam CT protocols in maxillary sinus imaging: part I-an in vitro study. Dentomaxillofac Radiol 46:20160323

34. Al Abduwani J, ZilinSkiene L, Colley S, Ahmed S (2016) Cone beam CT paranasal sinuses versus standard multidetector and low dose multidetector CT studies. Am J Otolaryngol 37:59-64

35. Koivisto J, Kiljunen T, Kadesjö N, Shi XQ, Wolff J (2015) Effective radiation dose of a MSCT, two CBCT and one conventional radiography device in the ankle region. J Foot Ankle Res 8:8

36. Ludlow J, Ivanovic M (2014) Weightbearing CBCT, MDCT, and $2 \mathrm{D}$ imaging dosimetry of the foot and ankle. Int J Diagn Imaging $1: 2$

37. Pugmire BS, Shailam R, Sagar P, Liu B, Li X, Palmer WE, Huang AJ (2016) Initial clinical experience with extremity cone-beam $\mathrm{CT}$ of the foot and ankle in pediatric patients. Am J Roentgenol 206:431-435

38. Koskinen SK, Haapamäki VV, Salo J, Lindfors NC, Kortesniemi M, Seppälä L, Huang AJ (2013) CT arthrography of the wrist using a novel, mobile, dedicated extremity cone-beam CT (CBCT). Skeletal Radiol 42:649-657

39. de Charry C, Boutroy S, Ellouz R, Duboeuf F, Chapurlat R, Follet H, Pialat JB (2016) Clinical cone beam computed tomography compared to high-resolution peripheral computed tomography in the assessment of distal radius bone. Osteoporos Int 27:3073-3082

40. Koivisto J, Kiljunen T, Wolff J, Kortesniemi M (2013) Assessment of effective radiation dose of an extremity CBCT, MSCT and conventional $\mathrm{X}$ ray for knee area using MOSFET dosemeters. Radiat Prot Dosim 157:515-524

41. Bacher K, Mermuys K, Casselman J, Thierens H (2009) Evaluation of effective patient dose in paranasal sinus imaging: comparison of cone beam CT, digital tomosynthesis and multi slice CT. In: Dössel O, Schlegel WC (eds) World congress on medical physics and biomedical engineering, Munich, Germany. IFMBE proceedings, vol 25/3. Springer, Berlin

42. Koivisto J, Schulze D, Wolff J, Rottke D (2014) Effective dose assessment in the maxillofacial region using thermoluminescent (TLD) and metal oxide semiconductor field-effect transistor (MOSFET) dosemeters: a comparative study. Dentomaxillofac Radiol 43:20140202

43. Yoshizumi TT, Goodman PC, Frush DP, Nguyen G, Toncheva G, Sarder M, Barnes L (2007) Validation of metal oxide semiconductor field effect transistor technology for organ dose assessment during CT: comparison with thermoluminescent dosimetry. Am J Roentgenol 188:1332-1336

44. Butson M, Haque M, Smith L et al (2017) Practical time considerations for optically stimulated luminescent dosimetry (OSLD) in total body irradiation. Australas Phys Eng Sci Med 40:167-171

45. Scarboro SB, Cody D, Alvarez P et al (2015) Characterization of the nanoDot OSLD dosimeter in CT. Med Phys 42:1797-1807

46. American Association of Physicists in Medicine (2010) Report of AAPM Task Group 111: the future of CT dosimetry-comprehensive methodology for the evaluation of radiation dose in X-ray computed tomography. American Association of Physicists in Medicine, College Park

47. Boone JM, McNitt-Gray MF, Hernandez AM (2017) Monte Carlo basics for radiation dose assessment in diagnostic radiology. J Am Coll Radiol 14:793-794

48. Huda W, Gkanatsios N (1998) Radiation dosimetry for extremity radiographs. Health Phys 75:492-499

49. Hart D, Wall BF (2002) Radiation exposure of the UK population from medical and dental X-ray examinations; report NRPB-W4; National Radiological Protection Board, UK

50. Neubauer J, Benndorf M, Reidelbach C et al (2016) Comparison of diagnostic accuracy of radiation dose-equivalent radiography, 
multidetector computed tomography and cone beam computed tomography for fractures of adult cadaveric wrists. PLoS ONE 11:e0164859

51. Nardi C, Taliani GG, Castellani A, De Falco L, Selvi V, Calistri L (2017) Repetition of examination due to motion artifacts in horizontal cone beam CT: comparison among three different kinds of head support. J Int Soc Prevent Commun Dent 7:208-213

52. Spin-Neto R, Matzen LH, Schropp L, Gotfredsen E, Wenzel A (2015) Factors affecting patient movement and re-exposure in cone beam computed tomography examination. Oral Surg Oral Med Oral Pathol Oral Radiol 119:572-578

53. LeQuire AK, Cunningham CJ, Pelleu GB Jr (1977) Radiographic interpretation of experimentally produced osseous lesions of the human mandible. J Endod 3:274-276

54. Vanhoenacker FM, Bernaerts A, Gielen J, Schepens E, De Schepper AM (2002) Trauma of the pediatric ankle and foot. JBR-BTR $85: 212-218$

55. Osgood GM, Thawait GK, Hafezi-Nejad N et al (2017) Image quality of cone beam computed tomography for evaluation of extremity fractures in the presence of metal hardware: visual grading characteristics analysis. Br J Radiol 90:20160539

56. Lang H, Neubauer J, Fritz B, Spira EM, Strube J, Langer M, Kotter E (2016) A retrospective, semi-quantitative image quality analysis of cone beam computed tomography (CBCT) and MSCT in the diagnosis of distal radius fractures. Eur Radiol 26:4551-4561

57. Vetter SY, Euler F, von Recum J, Wendl K, Grützner PA, Franke J (2016) Impact of intraoperative cone beam computed tomography on reduction quality and implant position in treatment of tibial plafond fractures. Foot Ankle Int 37:977-982

58. Faccioli N, Foti G, Barillari M, Atzei A, Mucelli RP (2010) Finger fractures imaging: accuracy of cone-beam computed tomography and multislice computed tomography. Skeletal Radiol 39:1087-1095
59. Nickoloff EL, Dutta AK, Lu ZF (2003) Influence of phantom diameter, $\mathrm{kVp}$ and scan mode upon computed tomography dose index. Med Phys 30:395-402

60. Siegel MJ, Schmidt B, Bradley D, Suess C, Hildebolt C (2004) Radiation dose and image quality in pediatric CT: effect of technical factors and phantom size and shape. Radiology 233:515-522

61. DeMarco JJ, Cagnon CH, Cody DD et al (2007) Estimating radiation doses from multidetector CT using Monte Carlo simulations: effects of different size voxelized patient models on magnitudes of organ and effective dose. Phys Med Biol 52:2583-2597

62. Turner AC, Zankl M, DeMarco JJ et al (2010) The feasibility of a scanner-independent technique to estimate organ dose from MDCT scans: using CTDIvol to account for differences between scanners. Med Phys 37:1816-1825

63. Tschauner S, Marterer R, Nagy E et al (2017) Surface radiation dose comparison of a dedicated extremity cone beam computed tomography (CBCT) device and a multidetector computed tomography (MDCT) machine in pediatric ankle and wrist phantoms. PLoS ONE 12:e0178747

64. Li X, Samei E, Segars WP, Sturgeon GM, Colsher JG, Frush DP (2011) Patient-specific radiation dose and cancer risk for pediatric chest CT. Radiology 259:862-874

65. Martin CJ (2007) Effective dose: how should it be applied to medical exposures? Br J Radiol 80:639-647

66. Lee C, Flynn MJ, Judy PF, Cody DD, Bolch WE, Kruger RL (2017) Body size-specific organ and effective doses of chest CT screening examinations of the national lung screening trial. Am J Roentgenol 208:1082-1088

67. Newman B, Ganguly A, Kim JE, Robinson T (2012) Comparison of different methods of calculating CT radiation effective dose in children. Am J Roentgenol 199:232-239 